\title{
An analysis on potential renewable energy system using triangular tile pasting as a Markov Chain in terms of Maximal Clique
}

\author{
Francis Anitha Florence Vinola ${ }^{1} \cdot$ Govindan Padma ${ }^{1}$
}

Received: 20 July 2021 / Accepted: 3 October 2021 / Published online: 5 February 2022

(c) King Abdulaziz City for Science and Technology 2022

\begin{abstract}
Transformation is an art of wavering chattels from one location to the other location or one zone to the other zone or one pattern to the other pattern. Designing any regular or irregular polygonal structures especially in tile pasting plays decisive portrayal of procreation. Tile pasting is a technique of gluing tiles in sequence of specific nature, and subsidize collection of structures to the constructors. The sequence of tile pasting can be analyzed and the best sequential path will be determined using Viterbi Algorithm. The pictorial representation of Viterbi algorithm is the trellis path that hand out more strength to the algorithm which shows the sequential order of tile pasting effectively. This paper will also be initiated to find the renewable energy produced by gluing triangular tiles of different polygonal structures and gives an intention to identify the most effective structure among the shapes discussed.
\end{abstract}

Keywords Markov chain · Triangular tile pasting · Viterbi trellis diagram · State diagram · Polygonal structures .

Renewable energy

\section{Introduction}

A model can be generated in two-dimensional geometry by pasting system which generates a tiling patterns includes pasting of square tiles, triangular tiles, Wang tiles etc. Tile pasting has a specific rule and it generates patterns such as square, hexagon, octagon and triangle etc. have been discussed in (Bhuvaneswari et al. 2016; Subramanian et al. 2009; Vaithyasubramanian et al. 2015). Pasting rule can be applicable in array token Petri Nets when the input places contain the same array as tokens have been discussed in Lalitha 2015a. Pasting system has been introduced using parallel array token Petri Nets in Lalitha and Rangarajan (2011). The tokens which are used in the colored Petri Net structures are not similar and different arrays have been used as a token in Lalitha 2015 b.

Markov process has its own properties which are discussed using the transition probability matrix (tpm) and the

Francis Anitha Florence Vinola

anithaflorence1981@gmail.com

Govindan Padma

govindanpadma1970@gmail.com

1 Department of Mathematics, Sathyabama Institute of Science and Technology, Chennai 119, India corresponding state transition diagram (Cinlar 2013). One of the powerful algorithm called Belief propagation algorithm were used in error correcting codes such as Low Density Parity Check Codes (LDPC) and TURBO codes (Padma 2014). An error can be identified and detected using the maximal cliques of an undirected graphical representation of a Markov random Field (MRF) which develops an idea of relating the graphical representation of Markov Chain properties in different aspects. One aspect is the Triangular Tile Pasting (TTP) using Trellis Diagram which was discussed in coding and de-coding signals. Trellis Diagram is the graphical representation of Viterbi Algorithm. Viterbi algorithm were used to identify the sequence alignment of human cells have been discussed in (Padma and Vijayalakshmi 2011; Özbay and Çekli 2018). Tile pasting of hexagonal and square tiling have been discussed and the speed of the molecule movement from one position to the other position, initial position of the molecules gets drastic changes due to the probability parameter $p$ were calculated using first passage time and first recurrence time (Saiguran et al. 2019).

Past few years' tremendous changes happened in the field of production, technology development, mobile communication and wireless communication etc. Though the productions of gadgets are more, the users and the awareness were comparatively less before COVID-19. Due to the lockdown 
and to overcome the pandemic situation, all corners have started using wireless communication under the Universe. Web users facing a problem to sustain secured passwords for transaction and in mobility, road managements are the massive bickering in present scenario. Since communication needs security, more research works carried out in the field of secured communication. $3 \times 3$ array password generations have been implemented to create a secured password in the field of Petri net theory (Vaithyasubramanian et al. 2015). Markovian Vehicular Channel Model (MVCM) has been proposed to serve effectively in Vehicular Ad Hoc Networks (VANETs) (Fazio et al. 2015). A Markov chain model has been adopted to fulfill the requirements of Request to Send / Clear to Send (RTS/CTS) covenant in procuring Mobile Ad Hoc Networks (MANETs) communication (Alnaghes and Gebali 2015). In multicast mobile Ad Hoc networks, Markovian model has been imposed to discriminate the packet propagation process at source and the fastest reception process at destination (Yang et al. 2019).

Energy- gives the strength, power and confident to overcome the problems faced by the human beings and nonhuman beings lives under the universe. Though energy is produced from various sources as a usage is more it becomes inadequate. So the recent researches are mainly focused on the resources which generate energy. The different types of energy called electrical energy, heat energy and thermal energy are produced from different sources like water, nuclear atoms and human bodies etc. The heat energy produced during ceramic tile production can be used as thermoelectric generators which will be converted into an electrical energy for disparate prospect. It has been portrayed by thermoelectric cooler modeling contains p-type and n-type semiconductors (Mohammadi 2018). Electrical energy can be produced by applying some weights on tiles pasted on the floor when piezoelectric sensors fixed on the tiles. Energy production depends on different phenomenon of piezoelectric sensors and the arrangement of tiles too. The molecular structures of ferroelectric materials align as it would be and separate the local charges (Kumar and Chaturvedi 2014). Human body contains more of proteins which act as a resourcing agent of energy for the body. The energy distribution in proteins and energy released during the peptide bonds were discussed using probabilistic Markov Random Fields (MRFs) and the functional relationship between Bayesian Networks (BNs) and MRFs were also identified using factor graph in terms of maximal cliques instead of statistical models (Padma and Vijayalakshmi 2011).

In view of the related works for the potential renewable energy, how important to provide the variety of resources generates energy to meet the peoples' requirements and frame safe zone for their better survival in the modern technology. In this paper a model is generated (Triangular pyramid, Square, Hexagon and Octagon) using Triangular Tile
Pasting (TTP) for the analysis of the renewable energy. The state diagrams corresponding to the trellises are discussed in terms of cliques (Sub graph of undirected graphical structure in which every two distinct vertices are adjacent) to generate renewable energy. This paper is arranged in the following order as preliminary concepts leading with different structures in relation with trellis diagrams, corresponding state diagrams, comparison of polygonal structures with tpm and maximal cliques, Discussion and results for renewable energy process using TTP as a Markov chain and the conclusion.

\section{Preliminaries}

The following are the basic definitions used to understand the concept applied in the construction of polygonal structures using triangular tile pasting as a Markov chain.

\section{Markov chain}

A probabilistic stochastic model which is defined in discrete time has the states that depend on the just past and not on the other states.

$$
\begin{aligned}
& \text { (i.e.) } p\left\{X_{i+1}=a_{i+1} / X_{i}=a_{i}, X_{i-1}=a_{i-1}, \ldots, X_{0}=a_{0}\right\}=p\left\{X_{i+1}\right. \\
& \left.=a_{i+1} / X_{i}=a_{i}\right\}
\end{aligned}
$$

\section{Viterbi algorithm}

The Viterbi algorithm is a dynamic programming algorithm for finding the most likely sequence of hidden states called the Viterbi path that results in a sequence of observed events, especially in the context of Markov information sources and Hidden Markov Models (HMMs).

\section{Trellis diagram}

A Trellis is a graphical representation of Viterbi algorithm, whose nodes are ordered into vertical slices with each node at each time connected to at least one node at a later time. The earliest and latest times trellis has only one node. Trellises are used in encoders and decoders for communication theory and encryption. Cost- constrained Viterbi algorithm is used to solve the problems occurred in green cellular networks and designate various resources to generate renewable energy in solar base stations.

\section{Tiles required}

Table 1 describes the tiles required for the structure construction that can be obtained by rotating an isosceles 
Table 1 Tiles required for structure formation

\begin{tabular}{|c|l|l|}
\hline Name & Triangle & \multicolumn{1}{|c|}{ Description } \\
\hline & & $\begin{array}{l}\text { An isosceles triangle with two sides of equal } \\
\text { length (1 uwit) and the third side of length } \\
\sqrt{2} \text { units. }\end{array}$ \\
\hline $\mathrm{b}_{0}$ & & $\begin{array}{l}\text { Rotate the given triangle about } 180^{\circ} \text { upward } \\
\text { or downward direction }\end{array}$ \\
\hline $\mathrm{c}_{0}$ & & $\begin{array}{l}\text { Rotate the given triangle as about } 90^{\circ} \text { in an } \\
\text { anti-clockwise direction. }\end{array}$ \\
\hline & $\begin{array}{l}\text { Rotate the given triangle an about } 90^{\circ} \text { in } \\
\text { clockwise direction. }\end{array}$ \\
\hline
\end{tabular}

triangle with two sides of equal length (one unit) and the third side of length $\sqrt{2}$ units to clockwise, anti-clockwise, upward and downward direction about $90^{\circ}$ and $180^{\circ}$.

\section{Pasting rules}

Any materials can be glued or pasted together in such a way that the sides of same lengths pasted together gives the perfect shape. As the Trellis diagram involves nodes, the four different triangles $a_{o}, b_{o}, c_{o}, d_{o}$ are taken as the nodes $00,11,01,10$ and pasting triangles with one another represents the edges. Table 2 describes the pasting rules among the triangles $a_{o}, b_{o}, c_{o}, d_{o}$ for constructing a regular or irregular polygonal structures.

- As the Trellis diagram involves an input, 0 and 1 are taken as the inputs.

- Input 0 represents, pasting triangular tiles of equal sides.

- Input 1 represents, pasting triangular tiles of sides of length $\sqrt{2}$ units.

Either or the tile pasting started with the inputs 0 and 1 , the tile pasted in the successive steps depends only on the tile precedes it and none before that. Therefore the TTP possess the Markovian property.

\section{Algorithm for constructing triangular pyramid}
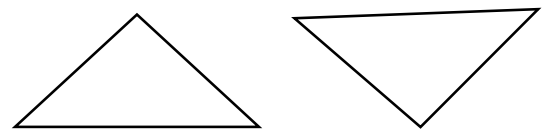

The following triangular pyramid is obtained from the triangles $a_{o}$ and $b_{o}$ using Viterbi Trellis Decoder algorithm.

Step 1: start with initial stage at triangle $\mathrm{a}_{\mathrm{o}}$.

Step 2: give input 1 , which represents the triangle $b_{0}$ can be pasted to the side of length $\sqrt{2}$ units with $a_{o}$ denoted in Red line.

Step 3: give input 0 , which represents the triangle $\mathrm{a}_{0}$ can be pasted to the sides of equal length (1unit) with $b_{0}$ denoted in black curve and go to step 2.

Step 4: steps 2 and 3 can be repeated and end with an input 0 to obtain the triangular pyramid (Figs. 1, 2, 3).

The state diagram having two states 00 and 11 which are connected to each other and no self loop shows that the different tiles $\mathrm{a}_{0}$ and $\mathrm{b}_{0}$ can be pasted together one after the other and the same tiles do not. Since the two distinct states of a state diagram for triangular pyramid are adjacent, it has a maximal clique of order 2 represents the Markov chain is irreducible (Anitha Florence Vinola and Padma 2020).

The maximal sequence path for constructing a triangular pyramid is given by the Trellis diagram which shows that the triangular pyramid structure should start with an input 1 and end with an input 0 .

\section{Algorithm for constructing square}
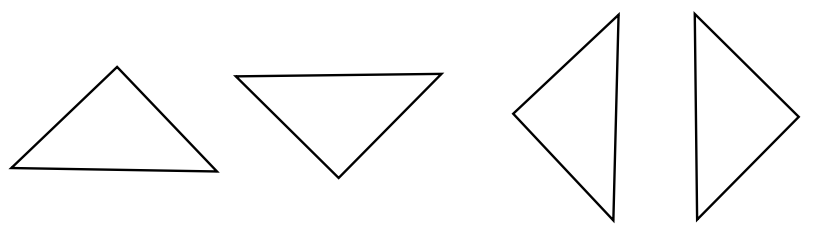
Table 2 Pasting rules for tiles

\begin{tabular}{|c|c|c|}
\hline Input & Description & Pasting positions \\
\hline $\begin{array}{c}00- \\
\mathrm{a}_{0}\end{array}$ & $\begin{array}{l}\text { Let } \mathrm{a}_{\mathrm{o}} \text { be an isosceles triangle } \\
\text { with two equal sides of } 1 \text { unit } \\
\text { and the third side of length } \\
\sqrt{2} \text { units. } \mathrm{b}_{0} \text { will be pasted } \\
\text { with the side of length } \sqrt{2} \\
\text { units of } \mathrm{a}_{0}, \mathrm{c}_{0} \text { will be pasted } \\
\text { in the right up of } \mathrm{a}_{0} \text { and } \mathrm{d}_{0} \\
\text { will be pasted in the left up of } \\
\mathrm{a}_{0} \text {. }\end{array}$ & \\
\hline $\begin{array}{c}11- \\
\mathrm{b}_{0}\end{array}$ & $\begin{array}{l}\text { Let } b_{0} \text { be an isosceles triangle } \\
\text { obtained by rotating } \mathrm{a}_{0} \text { about } \\
180^{\circ} \text { upward or downward } \\
\text { direction. } \mathrm{a}_{0} \text { will be pasted } \\
\text { above } \mathrm{a}_{0} \text { with the side of } \\
\text { length } \sqrt{2} \text { units, } \mathrm{c}_{0} \text { will be } \\
\text { pasted in the right down of } \mathrm{b}_{0} \\
\text { and } \mathrm{d}_{0} \text { will be pasted in the } \\
\text { left down of } \mathrm{b}_{0} \text {. }\end{array}$ & \\
\hline $\begin{array}{c}01- \\
\mathrm{c}_{0}\end{array}$ & $\begin{array}{l}\text { Let } c_{o} \text { be an isosceles triangle } \\
\text { obtained by rotating a about } \\
90^{\circ} \text { in anticlockwise } \\
\text { direction. } a_{o} \text { will be pasted in } \\
\text { the left down of } c_{o}, b_{o} \text { will be } \\
\text { pasted in the left up of } c_{o} \text {, and } \\
d_{o} \text { will be pasted in the right } \\
\text { side of } c_{o} \text { of length } \sqrt{2} \text { units. }\end{array}$ & \\
\hline $\begin{array}{c}10- \\
\mathrm{d}_{0}\end{array}$ & $\begin{array}{l}\text { Let } d_{o} \text { be an isosceles triangle } \\
\text { obtained by rotating a a about } \\
90^{\circ} \text { in clockwise direction. } a_{o} \\
\text { will be pasted in the right } \\
\text { down of } d_{o}, b_{o} \text { will be pasted } \\
\text { in the right up of } d_{o} \text { and } c_{o} \\
\text { will be pasted in the left of } d_{o} \\
\text { of length } \sqrt{2} \text { units. }\end{array}$ & \\
\hline
\end{tabular}

A single square can be obtained by pasting 4 triangular tiles $a_{0}, b_{o}, c_{o}$ and $d_{o}$ using Viterbi- Trellis decoder algorithm which starts with an input 0 and end with an input 0 .

Step 1: start with an isosceles triangle $\mathrm{a}_{\mathrm{o}}$.
Step 2: give input 0 to $\mathrm{a}_{\mathrm{o}}$, which is pasted to both $\mathrm{c}_{\mathrm{o}}$ and $\mathrm{d}_{\mathrm{o}}$ on either side of it.

Step 3: end the step by giving input 0 to both $\mathrm{c}_{\mathrm{o}}$ and $\mathrm{d}_{\mathrm{o}}$ will be pasted with single triangle $b_{0}$. 
Step 4: steps 1-3 can be repeated to fill the plane surface with square tiles (Figs. 4, 5, 6).

The state diagram for square structure having four states $00,11,01$ and 10 has a maximal clique of order 3 which is not connected to the other state leads to the Markov chain is reducible.

The maximal sequence path for constructing a square structure is well defined in the Trellis diagram which shows that the square should start with an input 0 and end with an input 0 .

\section{Algorithm for constructing hexagon}
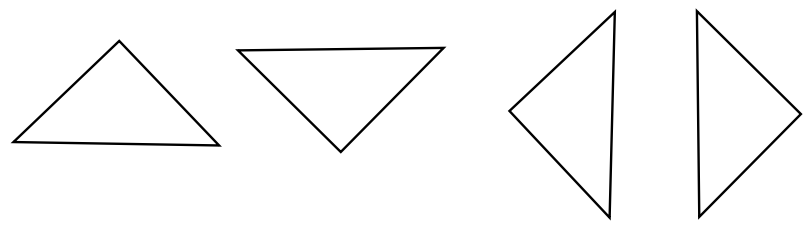

The hexagonal structure can be obtained by pasting four triangular tiles $\mathrm{a}_{\mathrm{o}}, \mathrm{b}_{\mathrm{o}}, \mathrm{c}_{\mathrm{o}}$ and $\mathrm{d}_{\mathrm{o}}$ using Viterbi- Trellis decoder algorithm which starts with an input 0 and end with input 1 .

Step 1: start with an isosceles triangle $\mathrm{a}_{\mathrm{o}}$.

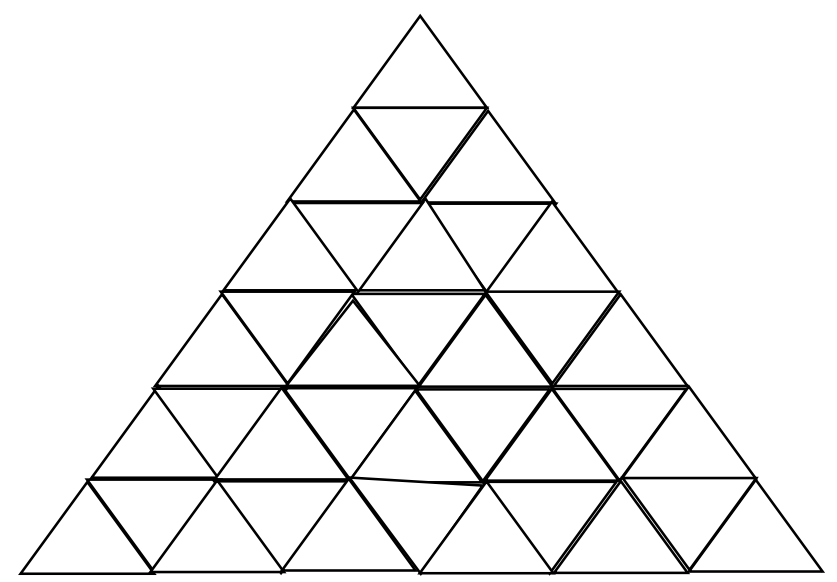

Fig. 1 Triangular pyramid filled with triangular tiles

$a_{0}$

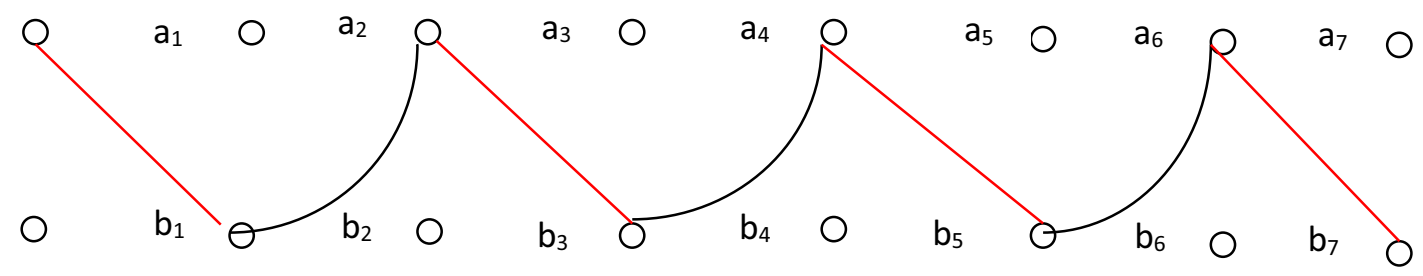

Step 2: give input 0 to $\mathrm{a}_{\mathrm{o}}$, which is pasted to both $\mathrm{c}_{\mathrm{o}}$ and $\mathrm{d}_{\mathrm{o}}$.

Step 3: give input 0 to both $\mathrm{c}_{\mathrm{o}}$ and $\mathrm{d}_{\mathrm{o}}$ will be pasted with single triangle $b_{o}$ which completes the square tile.

Step 4: give input 1 to both $\mathrm{c}_{\mathrm{o}}$ and $\mathrm{d}_{\mathrm{o}}$ will be pasted to $\mathrm{d}_{\mathrm{o}}$ and $\mathrm{c}_{\mathrm{o}}$ which completes the hexagonal tile (Figs. 7, 8, 9).

The state diagram for hexagon having four states 00,11 , 01 and 10 which are connected to each other and no self loop shows that the different tiles can be pasted together and the same tiles do not. Since the four distinct states of a state diagram for hexagon are adjacent, it has a maximal clique of order 4 represents the Markov chain is irreducible. Also the state diagram corresponding to hexagon in Fig. 9 is a complete graph which shows that the hexagonal structure is attractive and more efficient to generate the renewable energy.

The maximal sequence path for constructing an irregular Hexagon is given by the Trellis diagram which shows that the hexagon should start with an input 0 and end with an input 1.

\section{Algorithm for constructing octagon}
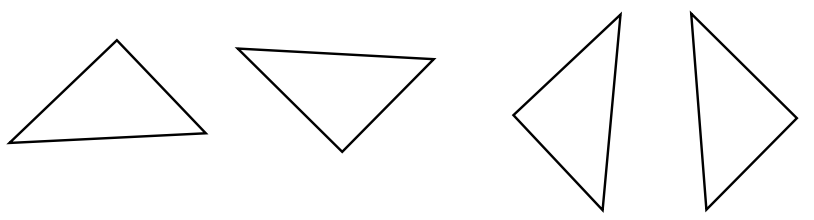

An Octagonal structure can be obtained by pasting 4 triangular tiles $\mathrm{a}_{\mathrm{o}}, \mathrm{b}_{\mathrm{o}}, \mathrm{c}_{\mathrm{o}}$ and $\mathrm{d}_{\mathrm{o}}$ using Viterbi- Trellis decoder algorithm which starts with an input 0 and end with input 0 .

Step 1: start with an isosceles triangle $\mathrm{a}_{\mathrm{o}}$.

Step 2: give input 0 to $\mathrm{a}_{\mathrm{o}}$, which is pasted to both $\mathrm{c}_{\mathrm{o}}$ and $\mathrm{d}_{\mathrm{o}}$.

Step 3: give input $1, \mathrm{c}_{\mathrm{o}}$ pasted to left of $\mathrm{d}_{\mathrm{o}}, \mathrm{d}_{\mathrm{o}}$ pasted to right of $c_{o}$ and $b_{o}$ pasted to down of $a_{o}$.

Step 4: give input 0, left up and right up of $c_{o}$ and $d_{o}$ pasted together with $b_{o}$, left up and down of $c_{o}$ pasted to $b_{o}$ and $a_{o}$, right up and down of $d_{o}$ pasted to $b_{o}$ and $a_{o}$, left down and right down of $b_{o}$ pasted to $d_{o}$ and $c_{o}$.

Fig. 2 Trellis diagram for triangular pyramid 


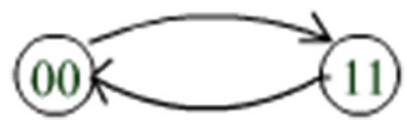

Fig. 3 State diagram for triangular pyramid

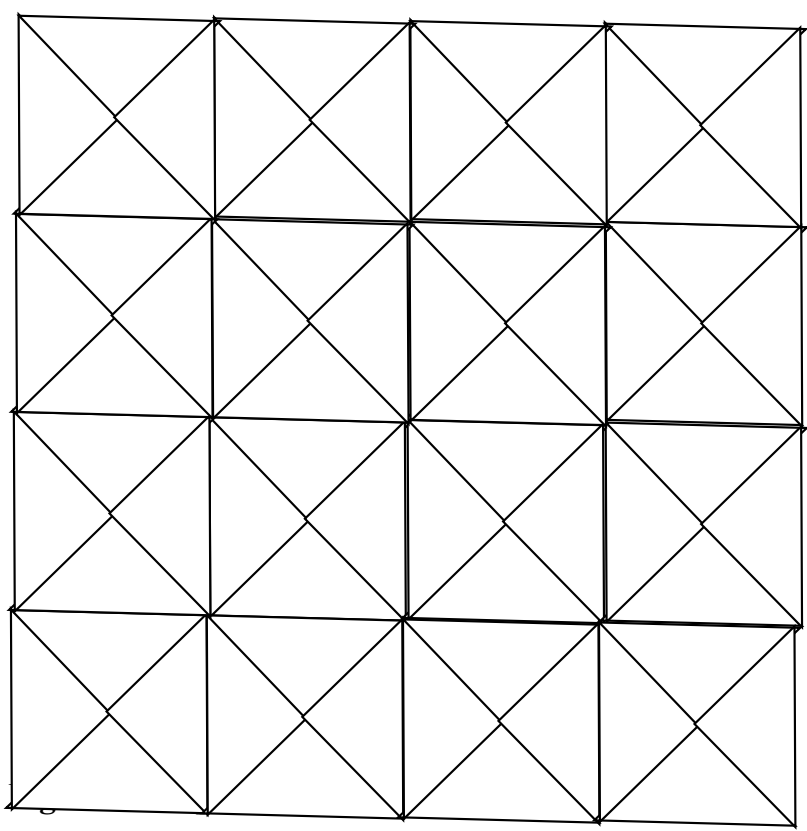

Fig. 4 Squares filled with Triangular Tiles
Step 5: give input $1, a_{o}$ pasted to up of $b_{o}, b_{o}$ pasted to down of $a_{0}, c_{o}$ pasted to left of $d_{o}$ and $d_{o}$ pasted to right of $c_{o}$.

Step 6: give input 0 to specific tiles, $d_{o}$ pasted to both $a_{o}$ and $b_{o}$ which completes the left cross, $c_{o}$ pasted to both $a_{o}$ and $b_{o}$ which completes the right cross, $a_{o}$ pasted to both $c_{o}$ and $d_{o}$ which completes the down cross and $b_{o}$ pasted to both $c_{o}$ and $d_{o}$ which completes the up cross of octagonal structure, $c_{o}$ pasted to right up of $a_{o}$ and $d_{o}$ pasted to left up of a completes an irregular polygon Octagon (Figs. 10,11).

The state diagram having four states $00,11,01$ and 10 which are connected to each other and no self loop shows that the different tiles can be pasted together and the same tiles do not. Since the four distinct states of a state diagram for octagon are adjacent, it has a maximal clique of order 4 represents the Markov chain is irreducible. Also the state diagram corresponding to octagon in Fig. 12 is a complete graph which shows that the octagonal structure is more attractive and efficient to generate the renewable energy.

The maximal sequence path for constructing an irregular Octagon is given by the Trellis diagram which shows that the octagon should start with an input 0 and end with an input 0 .

\section{Comparison of tpm with polygonal structures}

The tpm corresponding to the state diagrams that are drawn based on their respective trellises are differed by their number of zero elements and non- zero elements in "Algorithm for constructing triangular pyramid", "Algorithm for constructing square", "Algorithm for constructing hexagon", "Algorithm for constructing octagon" sections. In "Algorithm for constructing triangular pyramid" section, the odd

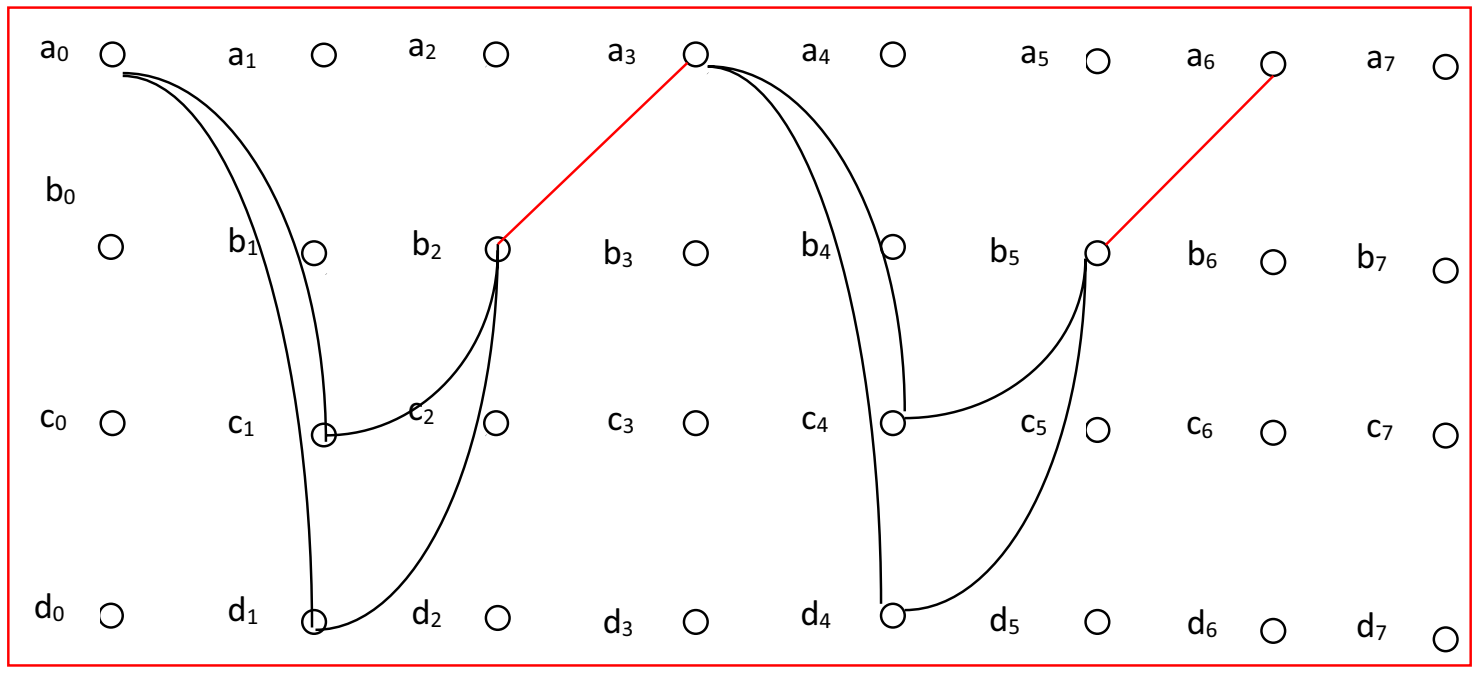

Fig. 5 Trellis diagram for square structure 


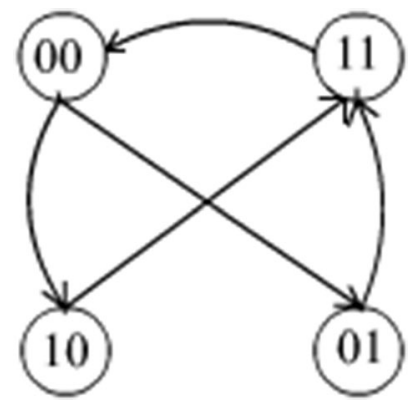

Fig. 6 State diagram for square structure

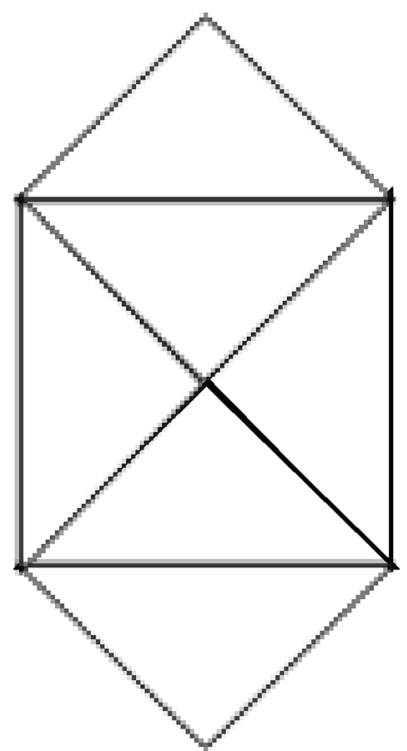

Fig. 7 Hexagon filled with Triangular Tile

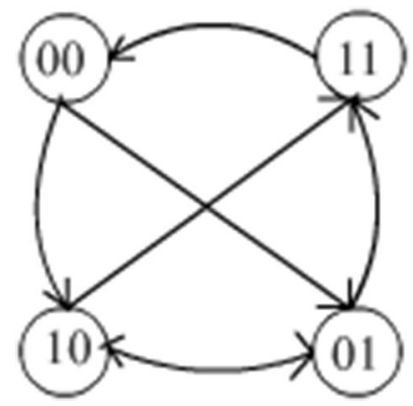

Fig. 9 State Diagram for Hexagon

powers $P^{3}, P^{5}, P^{7}, \ldots$ of the tpm resultant to the given tpm and the even powers $P^{2}, P^{4}, P^{6}, \ldots$ of the tpm resultant to the square of the given tpm says that the triangular pyramid formed by the triangular tiles pasted in alternate sequence. In Sect. Algorithm for constructing square, $P=P^{4}=P^{7}=, \ldots$ and $P^{2}=P^{5}=P^{8}=, \ldots$ and $P^{3}=P^{6}=P^{9}=, \ldots$

says that each square formed by the triangular tiles in every 3 steps. In Sect. Algorithm for constructing hexagon, the non-zero elements $p_{13}=p_{14}=p_{32}=p_{42}=\frac{1}{2}$ of the given tpm takes the same values in the higher powers of $\mathrm{P}$ from $P^{4}$ to $P^{6}$ and follows the pattern $p_{13}=p_{14}=p_{32}=p_{42}=\frac{4 N+1}{2^{n}}, N=1,2,3$ in the numerator and $n=$ Present power of $\mathrm{P}$ in the denominator. The non diagonal zero elements in the given tpm takes the same values in the higher powers of $\mathrm{P}$ from $P^{4}$ to $P^{6}$ and follows the pattern $p_{12}=p_{23}=p_{24}=p_{31}=p_{41}=\frac{4 N+1}{2^{n-1}}, N=0,1,2$ in the numerator and $n=$ present power of $\mathrm{P}$ in the denominator. The first two zero diagonal elements of the given tpm takes the same values in the higher powers of $\mathrm{P}$ from $P^{4}$ to $P^{6}$ and follows the pattern $p_{11}=p_{22}=\frac{4 N+1}{2^{n-2}}, N=0,0,1$ in the numerator and $\mathrm{n}=$ present power of $\mathrm{P}$ in the denominator. The last two zero diagonal elements of the given tpm takes

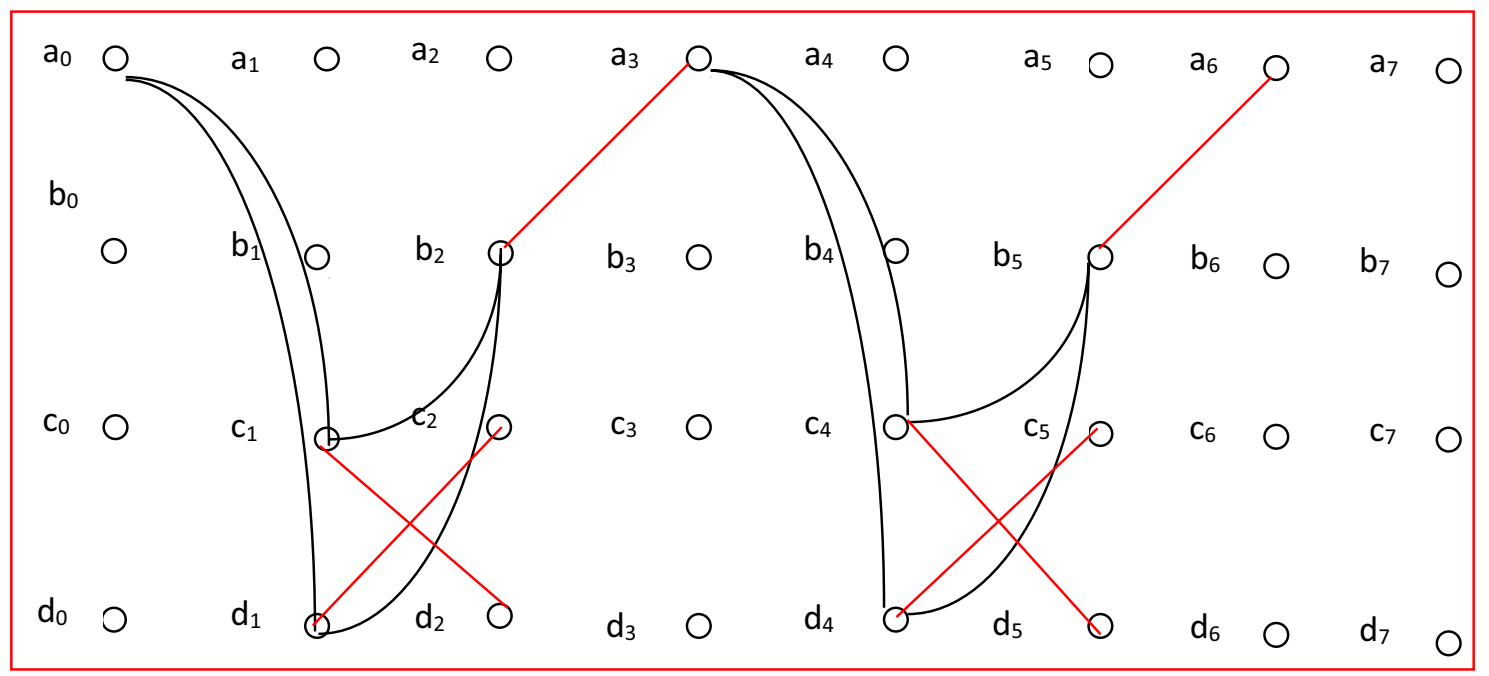

Fig. 8 Trellis Diagram for Hexagon 
the same values in the higher powers of $\mathrm{P}$ from $P^{4}$ to $P^{6}$ and follows the pattern $p_{33}=p_{44}=\left\{\begin{array}{l}\frac{x}{2^{n-2} 2^{2}}, \text { for even power of } P \\ \frac{x}{2^{n-2} 2}, \text { for odd power of } P\end{array}\right.$, $n=$ present power of $P$ and $x$ depends on the denominator. The sub diagonal non-zero elements in the given tpm takes the same values in the higher powers of $\mathrm{P}$ from $P^{4}$ to $P^{6}$ and follows the pattern $p_{34}=p_{43}$. Unit element $p_{21}=\frac{4 N+1}{2^{n-3}}$, $\mathrm{N}=0,0,0$ in the numerator and $\mathrm{n}=$ present power of $P$ in the denominator. The same pattern can be followed for the next three powers of $P$ from $P^{7}$ to $P^{9}$ with the numerator of

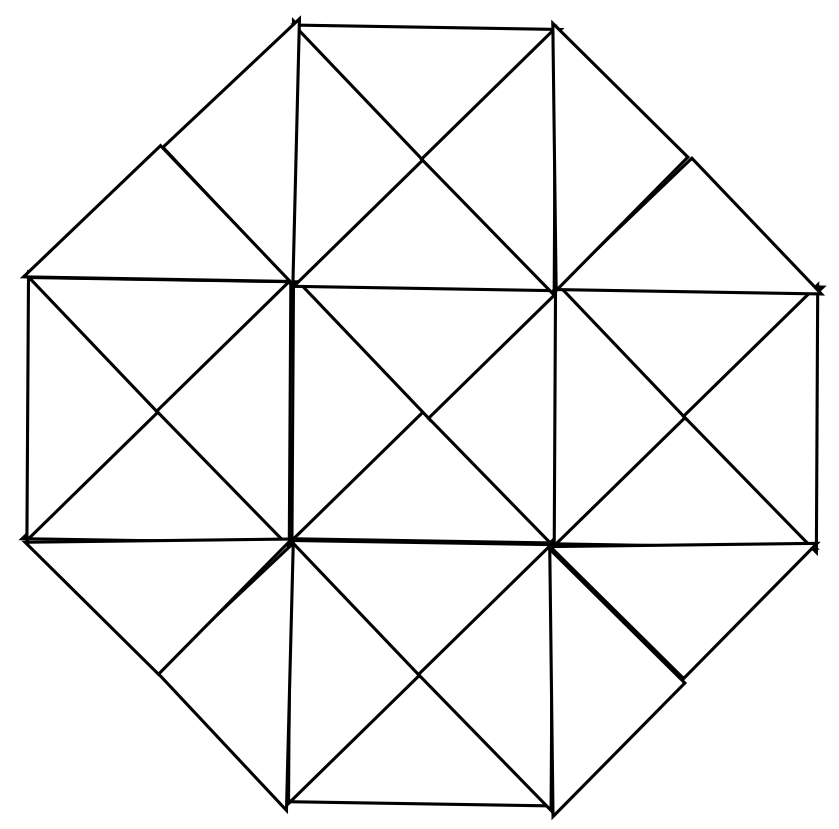

Fig. 10 Octagon filled with triangular tiles
$8 \mathrm{~N}+1$ and so on. The given tpm in Sect. Algorithm for constructing octagon shows that the Markov chain becomes irreducible in two steps. The non-zero elements in the given tpm whose higher powers follows the pattern $p_{i j},(i \neq j)=\left\{\frac{x}{3^{n}}, n=1,2,3,4,5, \ldots\right.$, where $x=1,2,7,20$, $61,182,547, .$. in which the first term $=1$, second term $=1 * 2$, third term $=(1+2) * 2+1$, fourth term $=(1+2+7) * 2$, fifth term $=(1+2+7+20) * 2+1$ and so on. The main diagonal elements $P_{i j}^{(n)}, i=j$ takes the same value as that of the nonzero elements $P_{i j}^{(n-1)}, i \neq j$ of the matrix which satisfies the Markovian property. An irregular polygonal structure hexagon is a combination of square and triangles. An octagon is a combination of square, hexagon and triangles. It has been discussed that every regular or irregular polygon which follows the Markovian property where the chain is irreducible. When a polygon is constructed with more number of irreducible Markov chains, then the polygon has a definite pattern. The pattern observed from the tpm along with the corresponding state diagram concludes that octagon is the powerful and attractive structure. Octagon creates lots of designs inside that structure and adds beauty for tiling region.

\section{Comparison of polygonal structures with maximal cliques}

From the "Algorithm for constructing hexagon" and "Algorithm for constructing octagon" sections, it is discussed that the polygonal structures hexagon and octagon has a maximal clique of order 4 and hence the Markov chain is irreducible. Therefore both hexagon and octagon has a definite pattern in TTP.

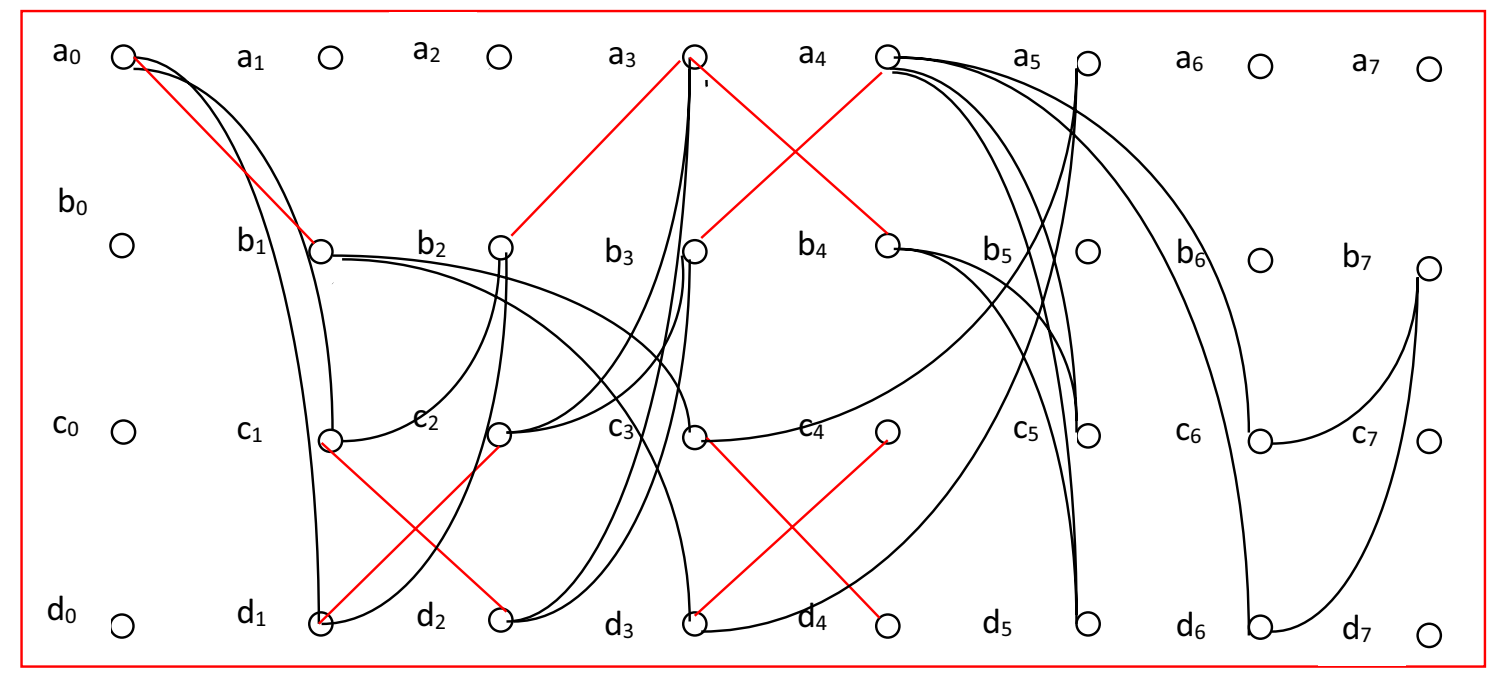

Fig. 11 Trellis diagram for octagon 
When the polygonal structures are discussed in terms of tpm corresponding to the state diagram octagon structure is more efficient. But in terms of clique hexagon and octagon are equally efficient.

\section{Discussion and results for renewable energy process using TTP as a Markov chain}

In the modern world technologist and industrialist have more responsibilities to provide the amenities in highest grade with affordable price. The policy of best from the waste

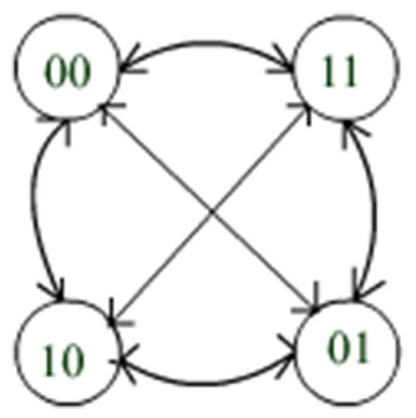

Fig. 12 State diagram for octagon

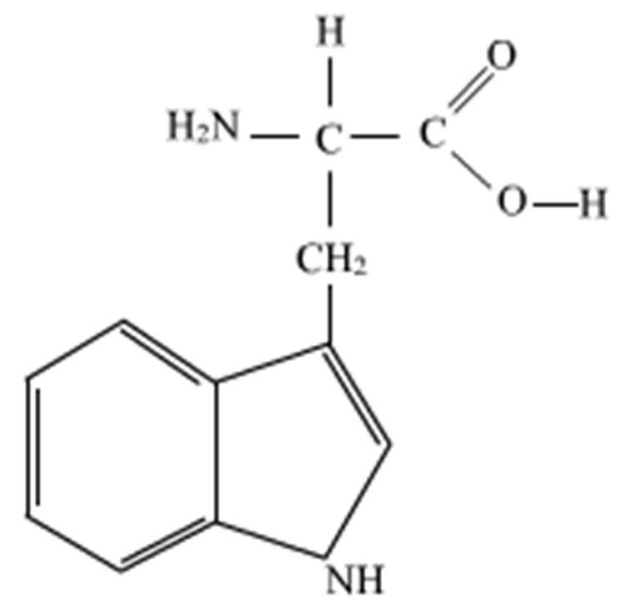

Fig. 13 Molecular structure of Tryptophan has reduced the pollution made from high level production materials. As production needs different types of energy, renewable energy is admissible in power generators. Photovoltaic generation assumed to be the one among the knock off renewable energy resources. Energy storage system deals with the multitudinous models for sustainability (Kim et al. 2021). Recent researches proved that the Markov chain models are adequate to assess the power system and the probabilities of their expected duration. When the Markov chain is irreducible, the system is intermittent and periodic (D’Amico et al. 2012). Size, shape and system pattern are paramount to salvage the renewable energy.

Triangular tile pasting (TTP) and extended triangular tile pasting system (ETTPS) have been discussed in pattern generation and P system (Bhuvaneswari and Kalyani 2018). This paper analyzes in detail the polygonal structures using TTP in terms of Markov chain. From Sects. 3, 5 and 6 it is concluded that the Triangular pyramid, hexagon and octagon are irreducible Markov chain. In the midst of polygonal structures discussed, hexagon and octagonal structures are assumed to sustain energy gained from different renewable energy resources. Free energy particles moving around the human body is taken as an example. Proteins are made up of amino acids and side chain. One of the most important aromatic amino acids is Tryptophan (Trp) contains $\alpha$ - amino group, $\alpha$ - Carboxyl acid group and a side chain with the molecular formula $\mathrm{C}_{11} \mathrm{H}_{12} \mathrm{~N}_{2} \mathrm{O}_{2}$ (Fig. 13).

The molecular structure of Tryptophan amino acid involves hexagon which could be related to an irreducible Markov chain based on the discussion made in Sect. Algorithm for constructing hexagon.

From Fig. 14, peptide bond formation of group of amino acids generates polypeptide chains during protein synthesis. Thermal energy has been released due to hydrolysis of peptide chain and $\mathrm{H}_{2} \mathrm{O}$ is separated from CONH. Energy availed is called free energy which is calculated using the formula $\mathrm{G}=\mathrm{E}-\mathrm{TH}$ and discussion made using factor graph (Padma and Vijayalakshmi 2011). Since the hexagonal structure has a clique of order 4 hexagon contains a cycle of even order implies that an assumed peptide chain is periodic in nature. Therefore the peptide chain formed using the group of amino acids is a Markov chain and also the chain is irreducible. As we concluded in Sect. Discussion and results for renewable
Fig. 14 Free energy released from peptide bond of Tryptophan

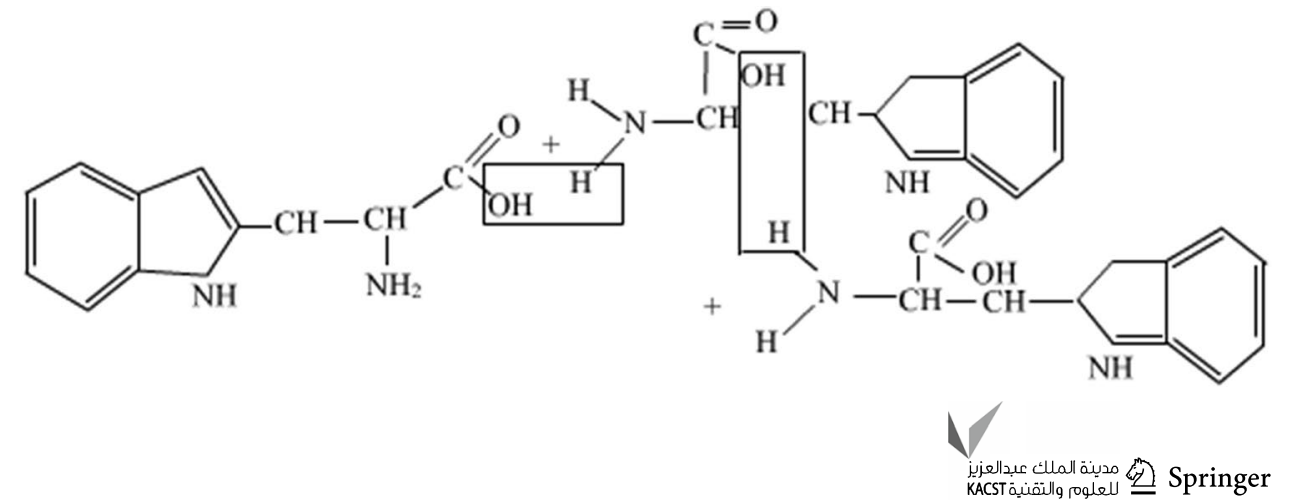


energy process using TTP as a Markov chain hexagon itself has a maximal clique of order 4, renewable energy obtained from the peptide bond formation of amino acids preserves hexagonal structure. Thus the minimal structure (hexagon) itself is convenient and efficient in bond formation of amino acids (Tryptophan) for renewable energy process. Renewable energy obtained by bond formation converted to Serotonin, a neurotransmitter prerequisite for monitoring appetite, sleep, frame of mind and the sickness. Tryptophan acts as a natural anodyne.

\section{Conclusion}

In this paper it is concluded that the TTP is a Markov chain. The polygonal structures triangular pyramid, square, hexagon and octagon have been discussed in terms of tpm and the corresponding state diagram and arrived that the octagonal structure is more effective than the other structures in TTP. Strong relationship between the irreducible Markov chain and structural designs in TTP is discussed in terms of cliques and concluded that both of the hexagon and octagon are equally efficient structures. This paper analyzes the generation of renewable energy which is considered as a Markov chain in terms of maximal clique for the amino acid (Tryptophan) bond formation. It is concluded that the hexagonal structure formed using TTP is sufficient for renewable energy resources in terms of maximal clique.

\section{References}

Alnaghes MS, Gebali F (2015) A Markov chain model for securing link layer in mobile Ad Hoc Networks. SAI Intelligent Systems Conference: 971-975

Anitha Florence Vinola F, Padma G (2020) A probabilistic stochastic model for analysis on the epileptic syndrome using speech synthesis and state space representation. Int J Speech Technol 23(2):355-360

Bhuvaneswari K, Kalyani T. (2018) Types of P system and triangular picture languages. IEEE Conference

Bhuvaneswari K, Kalyani T, Lalitha D (2016) Triangular tile pasting $\mathrm{P}$ system and array generating petri nets. Int J Pure Appl Math 107(1):111-128
Cinlar E (2013) Introduction to stochastic processes. 1st Edn. Elsevier: 416 (ISBN-10: 0486497976)

D'Amico G, Petroni F, Prattico F (2012) Reliability measures of second-order semi-Markov chain applied to wind energy production. J Renew Energy 2013:1-7

Fazio P, Tropea M, Sottile C, Lupia A (2015) Vehicular networking and channel modeling: a new markovian approach. IEEE Conference: 702-707

Kumar D, Chaturvedi P (2014) Piezoelectric energy harvesting from vibration induced deformation of floor tiles. National Power Electronics Conference (NPEC-2013), IIT, Kanpur: 1-6

Kim WS, Eom H, Kwon Y (2021) Optimal design of photovoltaic connected energy storage system using Markov chain models. Sustainability $13(7): 1-16$

Lalitha D (2015a) Rectangular array languages generated by a Petri Net. Comput Vision Robotics 332:17-27

Lalitha D (2015b) Rectangular array languages generated by a colored Petri net. In: Proceedings of IEEE International Conference on Electrical, Computer and communication technologies

Lalitha D, Rangarajan K (2011) Characterization of pasting system using array token Petri Nets. Int J Pure Appl Math 70(3):275-284

Mohammadi AM (2018) Renewable energy from thermal: electrical power generation in ceramic and tile industry. Innov Ener Res 7:212. https://doi.org/10.4172/2576-1463.1000212.Pp.1-7

Özbay B, Çekli S (2018) Power-efficient Viterbi decoder architecture and field programmable gate arrays Fpga implementation. Electrica 18(1):52-59

Padma G (2014) An analysis on the applications of Markov random fields in error correcting codes of nano memory cells. IEEE International Conference on Computational Intelligence and Computing Research: 1072-1075 (ISBN 978-1-4799-3972-5)

Padma G, Vijayalakshmi C (2011) A probabilistic approach for the analysis of free-energy distribution in proteins. Biotechnology 6(1):16-21

Saiguran M, Ring A, Ibrahim A (2019) Evaluation of Markov chains to describe movements on tiling. Open J Math Sci 3:358-381

Subramanian KG, Robinson T, Atulya KN (2009) Tile pasting P system model for pattern generation. Third Asian International conference on modelling and Simulation: 359-362

Vaithyasubramanian S, Christy A, Lalitha D (2015) Two factor authentication for secured login using array password engender by Petri net. Procedia Comput Sci 48:313-318

Yang B, Wu Z, Fan Y, Jiang X, Shen S (2019) Non-asymptotic capacity study in multicast mobile Ad Hoc Networks. IEEE Access $7: 115109-115121$

Publisher's Note Springer Nature remains neutral with regard to jurisdictional claims in published maps and institutional affiliations. 